\title{
LAS DEMANDAS SOCIALES Y EL ESTUDIO DE LOS MOVIMIENTOS SOCIALES
}

SOCIAL DEMANDS AND THE STUDY OF SOCIAL MOVEMENTS

Dr. Martín Retamozo (martin.retamozo@gmail.com) Centro de Investigaciones Socio-Históricas, Universidad Nacional de La Plata (Buenos Aires, Argentina)

\section{Abstract}

The concern of social sciences for mobilization and protests processes has resulted in the conformation of a peculiar field on social movements. Nevertheless, in spite of theoretical interest and intense methodological debate in reference to qualitative techniques, it is possible to say that discussion of these movements still remains open. In this perspective, according to Ernesto Laclau, this article suggests to identify social demands or the study of the conformation of social movements as political actors that dispute the social order. This implies a theoretical discussion but also an epistemological debate on the demands for the study of these movements.

Keywords: social movements, social demands, antagonism, protest, Laclau.

\section{Resumen}

La preocupación de las ciencias sociales por los procesos de movilización y protesta ha generado la conformación de un campo particular de estudio sobre movimientos sociales. No obstante, y a pesar tanto del interés teórico y del intenso debate metodológico en referencia a las técnicas cualitativas, es posible afirmar que la discusión sobre los ámbitos más relevantes para la investigación empírica de estos movimientos aún permanece abierta. Este artículo sugiere, siguiendo a Ernesto Laclau, identificar a las demandas sociales como claves para el estudio de la conformación de los movimientos sociales como actores políticos que disputan el orden social. Esto implica una discusión teórica pero también sobre las demandas sociales para el estudio de dichos movimientos.

Palabras clave: movimientos sociales, demandas sociales, antagonismo, protesta, Laclau.

De la memoria crecen resistencias/ agravios/daños/ padeceres/todo lo que el alma no puede perdonar Juan Gelman, Nota XI, Interrupciones I 


\section{Introducción}

Desde finales de la década del sesenta (1), el tema de los movimientos sociales ha constituido progresivamente un importante campo de estudio que concita la atención de diversas ciencias sociales. Este inusitado impulso se debió, en gran medida, a que fue por aquellos años cuando gran parte de las herramientas analíticas existentes se mostraron incapaces para dar respuestas rigurosas a los procesos históricos de movilización emergentes. Fueron las experiencias estudiantiles, feministas, pacifistas, entre otras, las que provocaron la necesidad de reflexionar desde nuevas ópticas sobre formas de acción colectiva y conflictos sociales que aparecían como novedosos si se comparaban con las maneras clásicas de contención política. Es así como surgieron, especialmente en el campo de la sociología política, un conjunto de teorías que proponían nuevos enfoques. La teoría de la movilización de recursos (McCarthy y Zald 1977, Craig 1994), la sociología de la acción de Touraine (1990 y 1991), el paradigma de la identidad (Melucci 1994 y 1999), los nuevos movimientos sociales (Lareña y Gusfield 1994), los estudios centrados en el proceso político (Tarrow 1997, McAdam, McCarthy y Zald 1999, McAdam, Tarrow y Tilly 2001), fueron algunos de los esfuerzos por avanzar en la comprensión de la movilización social y la acción colectiva en las últimas décadas. Más allá del análisis de los avances y limitaciones de cada uno de los intentos y las propuestas de diálogo inter paradigmático (Munck 1995) es preciso reconocer que la vasta literatura expuso con bastante claridad las diferentes posiciones teóricas ofreciendo caminos alternativos para la concepción de los movimientos sociales que, aunque no agota la discusión, la ordena bastante.

Sin embargo, en lo que se refiere a cuestiones epistemológicas el asunto no es tan claro (2), en especial porque en ocasiones encontramos un salto prematuro (que frecuentemente devela una enorme distancia) desde la discusión conceptual y teórica hacia el problema de las técnicas de investigación (cuantitativas y, especialmente cualitativas) (3) sin un abordaje de los campos epistémicos pertinentes para el estudio de los fenómenos de movilización social (4). Si bien de cada una de las teorías es posible reconstruir sus supuestos e implicancias epistemológicas -que pueden rastrearse principalmente en los estudios empíricos- hay un importante campo de discusión en ciernes en lo que respecta a los ámbitos de investigación pertinentes para abordar el estudio de los movimientos sociales (5). El presente artículo, por supuesto sin pretender agotar en medida alguna la discusión, se ubica en este intersticio con la intención de aportar al debate. Situados en un borde (o en un entremedio), procuramos recuperar algunos aspectos teóricos y epistemológicos de las demandas sociales como una piedra de toque en la investigación de los movimientos sociales. La dimensión teórica se plasma en la discusión de las categorías con las cuales desde diferentes enfoques se han conceptualizado a las demandas con el objeto de construir una perspectiva integradora. El planteo epistemológico, por su parte, se concreta en la identificación de los procesos asociados a las demandas que son factibles de ser construidos como anclajes de investigación. En este marco, nuestro objetivo específico consiste en exponer ciertos ejes que ubican a las demandas sociales como un terreno importante en términos teóricos y epistemológicos, factible de ser constituido como espacio mínimo de análisis y de investigación, e insuficientemente desarrollado por las teorías que guían el estudio de los movimientos sociales.

La tematización de las demandas sociales es fundamental para indagar en aspectos clave de la constitución de los movimientos. Esta relevancia es evidente si tenemos en cuenta que éstos elaboran demandas que presentan en el espacio público mediante acciones colectivas que sostienen en el tiempo y que implican procesos identitarios. Esto es reconocido explícitamente por algunas definiciones de los movimientos sociales y es supuesto (muchas veces implícitamente) por otras. No podemos -ni es el objetivo de este 
trabajo- discutir las prolíferas definiciones de movimientos sociales, nos bastará partir de un acuerdo mínimo: considerar que sea cual fuere el paradigma elegido dentro del esquema analítico hay un lugar para la demanda (6).

Guiados por la interrogación por las demandas sociales en el estudio de los movimientos, nuestra estrategia de exposición será la siguiente. En la primera parte proponemos reconstruir algunas de las nociones básicas de la teoría política contemporánea en referencia a la conformación del orden social. Esta sección inicial permitirá situar al lector en un universo conceptual que recupera aspectos teóricos para pensar el orden y el conflicto, los cuales serán relevantes para la epistemología de las demandas y permitirá sentar las bases para la discusión de la segunda parte, que busca problematizar el campo específico de las demandas sociales al considerarlo con potencialidad heurística. En la tercera sección, por su parte, exploraremos el vínculo entre demandas y movimientos sociales. Finalmente presentamos algunas reflexiones de orden epistémicometodológicas orientadas a identificar campos de investigación de las demandas.

\section{Orden político y demandas sociales}

Desde hace tiempo autores como Ernesto Laclau, Claude Lefort y Jacques Rancière, entre otros, vienen desarrollando reflexiones teóricas en torno a la constitución política de la sociedad y los procesos de conflictos que se suceden en torno a la conformación del orden social. A partir de estas contribuciones, nuestro objetivo en esta sección es incorporar categorías y movimientos conceptuales que permiten pensar las demandas en la perspectiva de aportar a la comprensión de los movimientos sociales.

Para pensar el proceso de constitución del orden social conviene distinguir analíticamente tres categorías: lo social, la sociedad y lo político. A lo social nos referiremos como un espacio indeterminado y potencialmente infinito de prácticas humanas sedimentadas. Autores como Laclau (2000:52) y Castoriadis (1986) han referido de diferentes maneras a un campo que excede el momento instituido de la sociedad (7). Lo social funciona como un terreno infinito, como un trasfondo donde se produce una operación que intenta poner cierto (imposible) orden finito. Este es el momento de lo político, donde mediante una operación hegemónica se sujetan sentidos, se detienen desplazamientos y se sobredetermina un espacio social dando lugar al orden social. Esa constitución del orden social ("la sociedad") es siempre fallida porque no todo "lo social" puede ser representado en "la sociedad", necesariamente hay un exceso que permanece fuera (como lo Real lacaniano se resiste a la simbolización) (Stravakakis 2007).

La primacía de lo político sobre lo social -una de las claves del pensamiento posfundacional (Marchart 2009)- debe entenderse, entonces, por la función de institución que el primero tiene sobre lo segundo. Autores como Lefort (1991) y Castoriadis (2007) han dedicado sus mejores reflexiones a los asuntos propios de esta distinción tan relevante aunque frecuentemente olvidada. Es en el momento de lo político donde se produce la sociedad mediante una operación hegemónica que ordena el plexo de lo social sedimentado para otorgarle un orden precario sobre la base de su propia contingencia. En efecto, hay una indeleble contingencia que sobrevuela y atraviesa al orden social aún cuando gran parte de la reproducción de un orden dado radique en el olvido de esa indeterminación originaria.

El recuerdo de la situación originaria es, precisamente, la apertura de ese territorio de lo político donde se hace presente la patencia de esa contingencia, como dice Zizek es "el momento de la apertura, de indecibilidad en el que se cuestiona el principio estructurante de la sociedad, la forma fundamental del pacto social" (1998:253). Este retorno es posible en tanto la constitución de un orden social se erige sobre 
una operación de exclusión de alternativas sociales (otros órdenes sociales) igualmente posibles. De este modo, el orden social debe entenderse como una construcción histórica, contingente y discursiva mediante una operación hegemónica. Para Laclau, el terreno de la constitución de la hegemonía es el discurso (Laclau 1985:23), es decir, que se requiere de una operación significante orientada a la articulación de elementos que entran en un juego relacional que los reconfigura. La centralidad de la categoría de "discurso" no debe hacer pensar en una forma de idealismo. La sociedad es discurso porque es una articulación de elementos, una ordenación particular de elementos. En este aspecto no hay que confundir discurso con actos de habla y escritura, la noción en Laclau tiene otro status teórico y epistemológico referido a ese conjunto de relaciones sociales y producciones de sentido que componen determinada sociedad. Esta noción de orden social como una articulación discursiva hegemónica de relaciones sociales será central a nuestros propósitos.

Ahora bien, es importante concebir que al interior del orden social, como resultado de esas relaciones sociales estructuradas mediante una operación hegemónica, se instituyen diferentes nombres y lugares (Rancière 1996). Dicho en otro registro, el orden es una multiplicidad de relaciones sociales parcialmente estructuradas y de muy diferente status que componen el mundo social, que lo reproducen y lo transforman. Y esta estructuración (que nace de un acto hegemónico indisociable del poder) produce en su interior diferencias, exclusiones, nombres, lugares, que se naturalizan históricamente pero que tienen el reverso de la contingencia. Estamos hablando de la producción-estructuración de la desigualdad, la asimetría, las relaciones de sometimiento y explotación que surgen como resultado de determinada ordenación social particular en la historia (8). La estructuración de la sociedad contiene así una pluralidad de diferencias (posiciones de sujeto al decir de Laclau y Mouffe 1985), algunas de las cuales implica definir lugares dominantes y por ende, a su reverso, subalternas. En este sentido, el orden social contemporáneo está atravesado por una multiplicidad de subordinaciones (como las de clase, de género, las étnicas, ecológicas). No obstante, esto no quiere decir que allí se erijan inmediatamente reclamos, actos de protesta, acciones o movilizaciones para acabar con esas situaciones o relaciones sociales, algo que significaría un tipo de mecanicismo (9). La identificación de posiciones subalternas (las víctimas, los dañados) nada nos dice aún de la capacidad de que esas relaciones sociales produzcan actores políticos. Laclau (1985:39) establece una sutil pero potente distinción entre subordinación y antagonismo que permitirá pensar el lugar a la construcción de demandas en los movimientos sociales

Laclau y Mouffe utilizan "subordinación” para referirse a la situación de determinadas posiciones de sujeto que están dominadas por otras (mujer/varón, homosexual/heterosexual, negro/blanco), mientras que reservan el término "opresión" para pensar el momento de resistencia en una situación de subordinación. Aquí las demandas emergen como un lugar de mediación entre una situación estructural de subordinación y la construcción de posibles antagonismos.

En una perspectiva convergente, Santos (2006) admite la multiplicidad de relaciones de subordinación pero identifica seis espacios estructurales donde pueden reconocerse formas distintas de poder y surgir allí diferentes demandas. De esta manera nos llama la atención sobre espacios, temporalidades y campos presentes en las formas de sociabilidad contemporánea: a) el espacio tiempo doméstico con su forma de poder patriarcal; b) el espacio tiempo de la producción con su forma de poder de explotación; c) el espacio tiempo de la comunidad donde el poder se plasma entre los que pertenecen a la comunidad y quienes no; $d$ ) el espacio estructural del mercado, donde la forma de poder es el fetichismo de la mercancía; e) el espacio tiempo de la ciudadanía, cuya forma de poder aparece en la relación vertical entre los ciudadanos y el 
Estado y f) el espacio tiempo mundial en cada sociedad que se vincula con el poder de un intercambio desigual entre países. Para nuestra discusión es extremadamente importante rescatar esos "espaciostiempos" que el autor identifica para pensar allí la elaboración de demandas sociales. Son estos campos (espacios-tiempos) los que constituyen nodos articulantes del orden social, espacios que pueden investigarse como terrenos de constitución de las demandas sociales, los antagonismos, las identidades y los movimientos sociales locales y globales.

En función de lo anterior, podemos comprender el vínculo conceptual entre demanda y antagonismo. Los antagonismos (10) son una producción originada en la identificación de una situación que tiene lugar al interior del orden social, la cual es considerada como injusta o indeseable y es presentada en el espacio público como una demanda insatisfecha. Esto no quiere decir que toda demanda sea antagónica. Laclau (2005b) repara en que las demandas "democráticas" muchas veces son solicitudes a la autoridad reconocida como competente sin necesidad de confrontación. No obstante, a partir de la elaboración de una demanda sobre una relación social de subordinación es posible construir espacios de antagonismo (para lo que resulta imprescindible definir un "otro"). Quién, por qué y cómo se produce la demanda que origina el antagonismo son asuntos para las cuáles aún nos falta caminar preguntando y para los cuales los conceptos de dislocación y subjetividad son claves.

Para Laclau el antagonismo designa una relación que no puede subsumirse bajo la noción de contradicción lógica ni de oposición real (Laclau 1985). Los antagonismos "no son relaciones objetivas sino relaciones que revelan los límites de toda objetividad" (Laclau y Mouffe 2004:14), son productos sociales emergentes de una acción de resistencia a los resultados de la estructuración de las relaciones sociales (Laclau 2000:25). Esta definición nos ayuda a pensar que el antagonismo erigido en determinadas relaciones sociales es una manifestación de recuerdo de la contingencia, la presencia del espectro de que todo puede ser diferente, la apertura de lo político aunque sea solamente una grieta en el orden social hegemónico. Allí la sutura que ordena parcialmente la estructura puede ser una fisura por la cual se cuela lo que Ernest Bloch llamó "todavía-no" del principio esperanza, es decir, esa apertura para potencialmente hacer posible lo que es imposible. La emergencia de antagonismos abre la posibilidad de la reconfiguración del orden social y son las huellas de la contingencia las que se levantan haciendo evidentes las limitantes de un intento de constituir la sociedad como algo pleno e idéntico. El antagonismo es la encarnación de un espectro que regresa para abrir lo político donde la política se muestra impotente, la demanda es su síntoma.

La elaboración de la demanda es un aspecto fundamental y lógicamente previo al antagonismo, aunque en el ámbito abierto del antagonismo pueden reelaborarse y producirse nuevas demandas. La demanda se produce en un intersticio del orden social como una "falta" y puede convertirse en vehículo de efectos dislocatorios, algo que dependerá de la capacidad de articulación de la demanda pero también del contenido (literal y su exceso metafórico). El estudio de la densidad de las demandas sociales permite también avanzar en uno de los puntos que más le preocupan a autores como Laclau y se vincula con la capacidad del sistema institucional para cancelar el conflicto y la posibilidad de las demandas de, mediante la articulación, interpelar aspectos del orden social para transformarlos (11).

Los procesos abiertos en momentos de elaboración de las demandas sociales no tienen ni un contenido ni una dirección prefijada a priori, eso es lo que hay que investigar en cada caso. Pero es la lógica de la demanda la que interpela al orden social (Naishtat 2004:369), a partir de significar una situación particular o una relación social como injusta, no deseable y posible de ser transformada. Esto no implica que de manera 
automática lo coloque en jaque, puesto que el orden social tiene sus mecanismos de absorción, cancelación o desplazamiento para intentar que el efecto de la demanda no afecten los centros neurálgicos del ordenamiento (12). Asimismo, los alcances de las demandas no pueden establecerse a priori, las "mismas" demandas en diferentes contextos pueden tener efectos muy distintos.

La contingencia e historicidad de las posiciones subordinadas y su potencial para -mediante la constitución de antagonismo- configurarse como opresivas, nos abre un campo de estudio fundamental para comprender los conflictos sociales y las movilizaciones colectivas. Esta posibilidad de nuevas decisiones que constituyan sujetos sólo puede pensarse en un espacio en que el orden social fracasa ( $y$, como vimos, el intento de cierre hegemónico siempre es fallido). No por esto hay que pensar que todas las estructuraciones sociales son semejantes. Puesto que estas son históricas, habrá algunas con mayor eficacia en sus operaciones de sutura. Pero todo orden es posible de entrar en crisis. Gramsci llamó crisis orgánicas a esos fracasos de los sectores dominantes por extender el consenso a los otros grupos sociales, Laclau radicaliza la noción en el concepto de "dislocación" para hablar de esos momentos donde la estructura deja mayores espacios a la libertad: ya no decide por todos en todo momento, sino que sus grietas son campos decisionales (13). Los sujetos, en lugar de condenados a la repetición, pueden abrir la diferencia, constituirse y expandir los efectos dislocatorios (14).

La lógica de la conformación de los sujetos sociales en estas condiciones dista mucho de ser lineal, por lo tanto poco se ganaría con proponer un esquema del comportamiento general. No podemos aquí detenernos en una teoría de los sujetos sociales (15), nuestro objetivo, más acotado, es proponer espacios de estudio para el análisis de los conflictos a partir de las demandas sociales. Esto supone analizar las formas de libertad abiertas en la dislocación y avanzar en comprender ésta no surge de la nada, ni como un efecto aleatorio por lo fallido del cierre hegemónico. Si el orden contiene de alguna manera al conflicto y está marcado (y cruzado) por la represión de alternativas, es necesario pensar cómo este exterior contenido regresa, cómo este retorno de lo reprimido constituye un proceso histórico, de lucha y donde las subjetividades y los sujetos tienen un lugar. Nos ubicamos así en el terreno de la relación sujetos sociales, subjetividad política y orden social, en especial para comprender el paso de la subordinación al antagonismo mediado por las demandas.

Allí es dónde el punto de llegada teórico de las reflexiones que seguimos a partir de Laclau, es el punto de partida para la investigación y las exploraciones centrales que pretendemos en este trabajo. ¿Cómo se produce el paso de una relación social de subordinación al antagonismo?, o dicho de otra manera: ¿cuál es el proceso de conformación de las demandas sociales?, ¿cómo se vinculan las demandas sociales a la formación de actores, identidades y sujetos?, ¿cómo es posible pensar la relación entre demandas sociales y orden social?, ¿cómo investigar las demandas sociales?

\section{Demandas sociales: sujetos y movimientos}

El abordaje teórico de las demandas sociales es factible de realizarse desde diferentes plataformas, las cuales pueden ser integradas gracias a que ofrecen ángulos analíticos complementarios. En una primera aproximación, la demanda puede ser entendida, siguiendo a Laclau (2005), al menos en dos acepciones: como petición y como reclamo. La petición se asemeja a la solicitud que alguien elabora sobre un asunto hacia la autoridad que considera competente (16). Allí, en principio, puede no haber beligerancia, no obstante esa demanda puede adquirir estatus de reclamo y por lo tanto una interpelación imperativa de un agente hacia otro para satisfacer la solicitud (17). 
Desde una segunda perspectiva, el estudio de las demandas tiene una dimensión que es iluminada desde el psicoanálisis. En esta orientación Frederic Jameson (1995) en referencia al esquema "L" de Jacques Lacan distingue entre la "necesidad" como hecho puramente biológico y la "demanda" que indefectiblemente está mediada por el lenguaje. En este plano (descartando el acceso a un hecho biológico puro o a una necesidad objetiva), se abre un importante campo de estudio sobre los discursos que constituyen la demanda. El abordaje de la constitución discursiva de esa "falta" que se vincula con el deseo (18) conduce, como dice Castoriadis (2007), a reubicar la importancia de los imaginarios sociales. Los elementos que intervienen en la construcción de la demanda tienen un carácter productivo de una nueva situación que además instituye un umbral para la acción.

Pero además es necesario ubicar la demanda en un tercer registro, esta vez en clave filosófica, como el espacio propio de la lucha por el reconocimiento. Si, como dice Hegel en la Fenomenología del Espíritu: "la autoconciencia es en y para si en cuanto que y porque es en sí y para sí para otra autoconciencia; es decir solo en cuanto se la reconoce" (1992:113), entonces en ese deseo elaborado como demanda encontramos las huellas del paso de la subjetividad al sujeto. En otras palabras, la demanda se orienta hacia el otro, hacia su reconocimiento, interpelando de alguna manera a la alteridad. Si traducimos en clave colectiva, podemos afirmar que en la construcción de la demanda social se encuentra inscripta una solicitud hacia otro (frecuentemente el sistema político) que lleva aquellos que referimos al principio: un pedido o reclamo.

Estas tres dimensiones de la demanda nos ayudan a pensar la constitución de los movimientos sociales. La identificación de una situación como injusta, la elaboración del pedido-reclamo, la interpelación de las alteridades y la lucha por el reconocimiento son aspectos que indudablemente ligan a las demandas con los movimientos.

Subjetividad y demandas sociales

La construcción de la demanda en los tres registros (petición y reclamo, deseo y reconocimiento) suponen necesariamente la injerencia de la subjetividad. En su elaboración como petición-reclamo los procesos subjetivos juegan un papel fundamental en la posibilidad de identificar-significar una relación social o una situación particular como factible de ser llevada al espacio público (19). Entendemos por subjetividad colectiva, siguiendo a Enrique de la Garza (2001) la configuración y articulación de códigos de significados (presentes en la cultura) para dar (construir) sentido a una situación (interpretación). En este plano podemos considerar que es la subjetividad la que configura diferentes significados y dota de un sentido particular a la relación social para hacerla eje de un reclamo que se considera legítimo. De esta manera, tal como recupera Jameson, la construcción de las demandas es algo que no puede pensarse independientemente del lenguaje -de la articulación discursiva-que produce o inscribe una situación como una demanda-deseo. Dicho en otros términos, es el sentido que se asigna a determinada situación la que la produce como demanda (y esta producción es subjetiva). Esto produce que un sector de la población decida actuar para buscar modificar esa situación percibida como injusta, sentida -parafraseando a Rancière (1996) - como un daño (20).

La construcción de la demanda involucra la intervención de la subjetividad colectiva para operar sobre una relación social particular y significarla como lugar de un antagonismo. Esta construcción de la demanda es eminentemente social y, en el plano que nos interesa, el proceso de construcción subjetiva es colectivo. Conviene aquí precisar que la referencia a la subjetividad colectiva no implica una especie de subjetivismo, sino la necesidad de pensar en las configuraciones de sentidos colectivos articulados para hacer inteligible 
(significativa) una situación de una determinada manera. En este aspecto la subjetividad colectiva es un proceso histórico-social factible de ser reconstruido mediante investigaciones rigurosas que apuntan a indagar en los códigos de significados movilizados para dar sentido. Estos códigos son condensaciones significativas precariamente sujetadas a contenidos particulares y tienen un origen histórico. Asimismo no pueden analizarse atómicamente puesto que actúan discursivamente, produciendo diferencias, desplazamientos, metáforas, metonimias y al estar abiertos admiten ser redimensionados en el proceso de construcción de las configuraciones coyunturales.

No podemos disociar tampoco la construcción de la demanda de los sentidos históricos y las subjetividades involucradas para identificar una situación como demanda. Ni soslayar la importancia de la subjetividad y la intersubjetividad en la posibilidad de establecer un campo común con otras demandas, esto es, para la articulación "equivalencial" (Laclau 2005) y la expansión de la demanda hasta involucrar a otros actores sociales. La factibilidad de extender sentidos compartidos y conformar subjetividades colectivas es una clave para indagar la formación de movimientos sociales, porque permite investigar las lógicas de extensión y amalgama de las demandas hasta formar conglomerados constitutivos de los movimientos.

La demanda pone en cuestión alguna relación social, saca al sujeto de su posición (21) y lo arroja a un terreno de constitución abierto por el corrimiento -y producción- de un nuevo lugar de enunciación. En este sentido, la demanda -análogamente a la decisión en el postestructuralismo- es un momento de la subjetividad antes del sujeto. Pero además, la elaboración de la demanda - con intervención de la subjetividad- permite construir un lugar de enunciación diferente al regulado por la repetición. Son los espacios de relativa autonomía los que operan para la construcción de campos de acción. El ejercicio de la acción tiene, en esta perspectiva, una función performativa del sujeto (Naishtat 2004:331), con lo que su análisis se vincula directamente a la comprensión de la conformación de la protesta y eventualmente de los movimientos sociales.

En vistas a lo anterior podemos destacar la necesidad de indagar en la lógica de la producción de las demandas sociales y los significados que la dotan de un sentido particular. Este sentido hace que la demanda sea construida como legítima y justa, por ende habilitante para la acción. Ambos términos (legítimo y justo) carecen de sentido positivo y sólo se vuelven significativos en un discurso que los incluye relacional y diferencialmente. Emerge aquí la importancia de los códigos, muchos de ellos binarios como dice Luhmann (1996), tales como justo/injusto, bueno/malo, correcto/incorrecto, moral/inmoral, etc., que son movilizados para dar sentido. Los sentidos colectivos convocados en cada elaboración de la demanda se convierten, como consecuencia, en un espacio analítico de relevancia los cuales pueden ser investigados de forma rigurosa. Lo mismo sucede con los procedimientos y lógicas utilizadas para las asignaciones de sentidos que nos acercan tanto a las teorizaciones sobre el sentido común y la vida cotidiana en autores como Antonio Gramsci, Alfred Schutz y Agnes Heller, como los espacios abiertos en experiencias extraordinarias que dislocan la cotidianeidad.

\section{Epistemología de las demandas sociales}

A partir de lo expuesto hasta aquí podemos identificar dos grandes dimensiones iniciales de investigación: la estructuración del orden social que produce en su interior cierta situación o relación social que luego se "demandiza" (su historicidad, posiciones de sujeto, articulación de tiempos y espacios, relación de los procesos globales y los locales, etc.) y la subjetividad (también su historicidad, su gramática, sus lógicas, contenidos, etc.) como instancia de producción de la demanda. Ambos espacios son fundamentales. La 
estructuración del orden social permite el análisis de las posiciones de sujeto y el reconocimiento de un conjunto de relaciones sociales posibles de entender como subordinación. No obstante, en tanto la demanda no es algo "objetivo" sino, repitámoslo, el síntoma de la falla de la objetividad misma, es necesario incorporar el campo vinculado a la subjetividad colectiva para indagar cuando una relación social de subordinación se construye como injusta y referencia de una demanda (22). Las demandas, en este sentido, condensan historicidades a la vez que son históricas. Castoriadis (2007:146-147) sugiere apuntes valiosos que en nuestra discusión se insertan en la revalorización de los sentidos legitimantes de la acción. En la construcción de lo que se puede demandar y aquello que no puede ser instalado como una demanda en un momento determinado. Como reverso de la demanda es importante indagar en la capacidad de las instituciones que gestionan el orden social para dar respuestas, definir, desplazar o cancelar el conflicto. Esto dirige nuestra atención hacia otro ámbito de investigación, además del contenido de la demanda (tanto literal como su exceso metafórico): la propia composición de las instituciones y su funcionamiento que pueden arrojar diversos resultados a la hora de procesar las demandas sociales (23).

La historicidad y la estructura de la demanda son terrenos que aunque frecuentemente descuidados, ameritan atención puesto que implica una entrada a la subjetividad colectiva. La construcción de la demanda está sobrecargada de sentidos históricos que fungen como su condición de posibilidad (y en determinadas ocasiones pueden ofrecernos pistas sobre sus limitaciones) y operan tanto en la producción del sujeto como en la apertura de campos de experiencia. En este aspecto consideramos relevante analizar el contenido de las demandas en tanto su composición puede ayudarnos a comprender la posibilidad o no del sistema institucional de cancelarla diferencialmente. En este camino conviene distinguir analíticamente el contenido literal de una demanda y su exceso metafórico (el que se vincula con su capacidad de tornarse tendencialmente vacío, como dice Laclau). En efecto, nos quedaríamos con un análisis sesgado si sostuviéramos que las demandas se agotan en su sentido literal. Las demandas, como construcciones discursivas, provocan desplazamientos metafóricos que pueden rearticular a otras demandas, por lo tanto la atención a los contenidos manifiestos de las demandas es necesario complementarla con el análisis de los excesos metafóricos. Precisamente, en la capacidad de ganar en vacuidad y situarse como significantes vacíos (Laclau 1996) (24) radica mucho de la posibilidad de pensar en los procesos mediante los cuales una determinada demanda es un nodo que amalgama a otras.

En otro lugar (Retamozo 2006b) hemos analizado la demanda de "trabajo" elaborada por el movimiento de desocupados en Argentina. Allí la exigencia de trabajo condensó una serie de otras demandas que excedían a la literalidad del término convirtiéndolo en una sinécdoque para referirse a una multiplicidad de reclamos de diferente índole que incluían: educación, salud, seguridad social, vivienda, certidumbre, identidad, futuro, etc. En este aspecto es posible a partir de la investigación empírica reconstruir el sintagma de la demanda, sus alcances y desplazamientos así como el entramado cultural sobre el que se apoya su construcción y el que puede proveer de ciertos sentidos para interpretar situaciones. También es conveniente incluir en el dispositivo analítico la consideración de las operaciones retóricas que constituyen un campo discursivo capaz de articular diferentes demandas. La retórica y sus tropos (como la metáfora, la metonimia, la sinécdoque, la catacresis, por ejemplo) constituyen importantes herramientas analíticas para la reconstrucción de la demandas

El estudio de las demandas sociales, las subjetividades colectivas articuladas y la conformación de los sujetos sociales se constituyen en claves analíticas para la investigación tanto de la formación de los movimientos sociales, como de la institución del orden social, su reproducción y su cambio. 


\section{Algunas consideraciones finales}

A partir de la discusión teórica, en este artículo se han ido esbozando dos grandes campos de estudio que necesariamente se cruzan: por un lado la conformación del orden social, por otro los procesos de configuración de la subjetividad colectiva que intervienen en la construcción de las demandas sociales. De allí se desprenden sendos espacios sobre los que es necesario construir la mirada epistemológica y la intervención metodológica.

La reconstrucción del orden social en que se desarrolla una movilización supone abordar las formas de estructuración de las relaciones sociales en los espacios concretos y de acuerdo al problema de investigación. El estudio de las relaciones sociales estructuradas en los campos económicos, culturales y políticos considerados ya como ineludibles puede ser complementado con la atención al menos a tres espacios. Por un lado, los procesos de institución del orden social que pueden observarse tanto en la lógica de articulación hegemónica (Laclau 2000 y 2005) como en los contenidos de los imaginarios colectivos que producen, eso que Castoriadis (1986) llamó el magma social. La atención a estas dimensiones convertidas en campos epistémicos requiere de construcciones metodológicas innovadoras y creativas centradas en la producción social de discursos, sentidos y significados. El análisis de los discursos sociales es de gran relevancia para dar cuenta de las disputas políticas, puesto que permite indagar las gramáticas de producción y recepción que operan a la hora de instalar la demanda por parte de los sujetos. En esta dimensión, el estudio de las formas en que los medios de comunicación masivos se vinculan con la producción de los significados sociales cobra cada vez mayor relevancia en las sociedades contemporáneas.

Los desafíos de investigación, de este modo, implican un esfuerzo de superación de las fronteras autoimpuestas por las diferentes ciencias sociales en cuanto a sus dominios epistemológicos y enfoques metodológicos. El abordaje del espacio de "lo político" a partir de estrategias de investigación social es tal vez uno de los pendientes más acuciantes en esta perspectiva. Este terreno ha sido dominado por la filosofía y la teoría política y poco abordado por las ciencias sociales, en parte debido al desafío epistémicometodológico que tal empresa significa.

En esta misma perspectiva, es relevante hacer objeto de estudio los espacios constituidos (las instituciones) que son cristalizaciones de significados, pero que adquieren una forma de operación sobre el orden social en lo que se refiere a su gestión, regulación y reproducción. La mirada sobre los andamiajes institucionales que gobiernan al orden social en sus diferentes niveles permitirá reconstruir campos de acción e interacciones entre actores, así como las posibilidades, potencialidades y limitaciones de interpelación de las demandas dentro de un contexto socio-histórico particular o "estructuras de oportunidades políticas" (Eisinger 1973, Tarrow 1997). La mirada sobre las instituciones ha sido dominada por versiones de la ciencia política proclamada neoinstitucionalista que las reducen a reglas de juego. Aquí, la sociología ha incursionado de manera mucho más eficaz, aunque reste avanzar en situar el análisis social de las instituciones.

Podemos identificar también un tercer campo de estudio en este plano referido al orden social a partir de los trabajos de Michel Foucault (1991). El estudio de los mecanismos de sujeción, los dispositivos y las dimensiones microfísicas del poder que reproducen el orden social requieren de una atención especial. Foucault llamó gubernamentalidad al conjunto de nuevas tecnologías del gobierno de las sociedades contemporáneas. El estudio de los procesos de sujeción-subjetivación, la producción de los dispositivos disciplinarios que incluyen y trascienden la estatalidad (las tácticas del gobierno), la elaboración de los discursos disciplinarios y las formas de poder presentes en las sociedades de control son algunos de los 
temas insoslayables que exigen esfuerzos de invención y creación epistemológica y, fundamentalmente, metodológica. Tarea esta sin dudas pendiente y que no estamos en condiciones de abordar aquí.

En una dirección compartida podemos mencionar la necesidad de tener en cuenta la existencia de los sujetos sociales que actúan en una sociedad determinada. Específicamente nos referimos a actores como los partidos políticos, los sindicatos, otros movimientos sociales, líderes, etc., que pueden ser fundamentales a la hora de elaborar una demanda o incidir en su receptividad. En efecto, la construcción de las demandas sociales muchas veces dependen de la existencia de estos factores o precisamente surgen allí donde estos actores no pueden ejercer su acción. En tanto la elaboración de una demanda es una producción social, resulta fundamental observar la presencia de sujetos que pueden moldear una demanda en determinadas circunstancias. Allí además hay lugar para los recursos involucrados en la elaboración de esa demanda, tal como indica la Teoría de la Movilización de Recursos.

Convergentemente es preciso atender a la otra gran dimensión para el estudio de las demandas sociales desde el enfoque que nos concierne. Nos referimos a la investigación de la constitución de la subjetividad colectiva y su intervención en la construcción-identificación-elaboración de la demanda. Este es un aspecto crucial y pone en evidencia la necesidad de la distinción entre subjetividad colectiva y sujeto social. Esto supone concebir un espacio analítico en la articulación de significados que construyen la demanda e instauran espacios de acción performativa del sujeto. De este modo los sujetos sociales serían producto de determinadas articulaciones subjetivas parcialmente estables que incorporan aspectos identitarios, dimensiones de reconocimiento, proyecto y acción. La atención a cómo se construye una subjetividad colectiva que permite elaborar una situación o relación social como demanda constituye otro de los desafíos metodológicos que requiere la atención de las técnicas cualitativas (25).

La propuesta aquí esbozada sobre indagar en las demandas sociales como campo relevante para el estudio de los movimientos sociales no significa en modo alguno que el estudio de éstos se agote allí. No obstante, a nuestro entender resultan claves analíticas relevantes tanto porque brindan un anclaje para el estudio de las subjetividades colectivas involucradas en la conformación de los movimientos sociales.

De lo que se trata es de ir progresivamente identificando terrenos de investigación que requieren a la vez que de desarrollos teóricos, de reflexiones epistemológicas orientadas a las dimensiones más concretas de la investigación empírica sobre los movimientos sociales. Es evidente que además se requiere un esfuerzo de integración conceptual que ponga en sintonía el estudio de las demandas con otros terrenos factibles de investigación en el tema de los movimientos sociales como lo son los imaginarios, los proyectos, las organizaciones y las acciones colectivas. Si aceptamos el complejo desafío de hacer investigación rigurosa y creativa, de calidad y asociada a los procesos políticos de las sociedades, tal vez aportemos a devolver a las ciencias sociales su lugar crítico en nuestro tiempo.

\section{Notas}

(1) Esto no significa en modo alguno que antes de esa etapa no existieran preocupaciones sobre el conflicto social y la acción colectiva, desde Hobbes a Marx, pasando por Hegel, desde los enfoques psico-sociológicos hasta el funcionalismo, cada intento de pensar lo político provocó de alguna manera la atención sobre aspectos de la acción, el conflicto y la movilización social. 
(2) Estas dimensiones, naturalmente, no se encuentren por completo fuera de la agenda de los estudios sobre movimientos sociales. Trabajos como los de Giménez 1994 y Schuster 2005 son contribuciones dignas de mención.

(3) Entre los trabajos más notables podemos destacar Santalamacchia, Colón y Rodríguez 1983, Santalamacchia 1987, Villansante 1994, entre otros.

(4) No obstante es preciso reconocer que en los estudios empíricos necesariamente se construyen lugares privilegiados en términos epistemológicos. Así, la Teoría de la Movilización de Recursos se focalizó en aspectos organizacionales y en los recursos disponibles, y la literatura centrada en el "Proceso Político" atendió también variables del sistema político llegando a incorporar aspectos culturales como los "marcos de significación" (McAdam 1994, Klandermans y Johnson 1995, Chihu 2000).

(5) El trabajo de Hugo Zemelman "Conocimiento y sujetos sociales" (1987) si bien no se ocupa específicamente de los movimientos sociales constituye una obra imprescindible para un epistemología de los movimientos y sujetos sociales. Para una discusión más detallada sobre esta perspectiva puede consultarse Retamozo 2006a y 2007.

(6) Esto puede constatarse incluso en las concepciones enfocadas al comportamiento colectivo como la Escuela de Chicago o los trabajos pioneros de Neil Smelser que hacían referencia a los agravios (deseos y expectativas) como una causa de la acción colectiva. Los estudios posteriores, centrados en la privación relativa (Ted Gurr) también repararon en el lugar relevante de las demandas en la explicación de la movilización social. En algunos enfoques contemporáneos esta presencia es explícita para definir un movimiento social (Schuster y Pereyra 2001:50), en otros la mirada sobre las reivindicaciones, los intereses y los conflictos se encuentran latente (Touraine 1990 y 1991, Tarrow 1997). No obstante la presencia de las demandas sociales, éstas no han sido un tópico lo suficientemente atendido por los estudiosos del tema.

(7) Ambos autores proponen distinciones analíticas en el plano ontológico que son fundamentales. No obstante, la interpretación que en este trabajo se defiende implica cierta distancia tanto con la consideración de Castoriadis de un espacio natural (pre-social) donde lo histórico- social (la sociedad) se "apoya"; como de Laclau, autor en el que podemos reconocer al menos tres usos de "lo social": a) como sinónimo de sociedad; b) como "prácticas sociales sedimentadas", naturalizadas al interior del orden social y que pueden "reactivarse"; c) como campo que excede lo simbólico (al orden social). Este tercer uso es el que privilegiamos en este trabajo.

(8) En los términos de Enrique Dussel: “todo sistema político (...) no puede ser nunca perfecto (sería necesario tiempo infinito, inteligencia y voluntad ilimitadas, etc.,) produce inevitablemente efectos negativos, en el mejor de los casos no intencionados" (2006:101), estos efectos negativos son sufridos por las víctimas del sistema (Dussel 1998).

(9) Autores clásicos como McCarthy y Zald repararon en la existencia de una multiplicidad de motivos de queja y que no todos cobran fuerza y visibilidad en el espacio público o concitan acción colectiva. Esto motivó la atención de los recursos para la movilización en torno a una queja.

(10) El debate sobre el status del antagonismo nos llevaría fuera de los límites del presente trabajo. No obstante, podemos indicar que hay al menos tres sentidos de antagonismo. Como límite de la objetividad un 
sentido ontológico en Laclau, como imposibilidad del sujeto pleno Zizek y la dimensión identitaria y como la reactivación de relaciones sociales que despiertan el espectro de lo político. Este tercer sentido es privilegiada en esta sección.

(11) Para una evaluación de la orientación política de las demandas y los movimientos sociales es necesario introducir un elemento que ha permanecido ausente en nuestro análisis: la dimensión ético-política de las demandas y de los movimientos. Una parte del problema, la acción de los sectores dominados, es iluminada por Jacques Rancière. Para el autor, como ya se dijo, la organización de la sociedad produce daño a una parte de la comunidad política. Ahora bien, para que se produzca es daño debe haber algo que sea dañado y eso dañado es el principio organizador de las sociedades modernas, una premisa de igualdad-libertad que legitima al orden social (Rancière 1996). En efecto, ese orden social que produce daños puede ser puesto en cuestión (mostrando su radical contingencia) por parte del pueblo que se muestra en desacuerdo con esa contradicción manifiesta entre la igualdad-libertad que legitima al orden y su negación en la vida social cotidiana. Esto es posible por la premisa de Rancière sobre "la igualdad última sobre la que descansa todo orden social" (1996:30) que permite un criterio de demarcación entre luchas genuinamente democráticasigualitarias y otros conflictos no basados en este principio. Es evidente que la concepción de un principio para el orden social conlleva los problemas propios de posturas universalistas. No obstante, es indudable que dicho principio -fundamental para la modernidad occidental- es constitutivo del imaginario democrático y tiene potencialidades emancipatorias.

(12) Como indica O’Donnell (1978) el Estado como aspecto de las relaciones sociales que conforma el orden social tiene esta función.

(13) Como acertadamente destaca Emilio de Ípola (2000), a pesar de la centralidad de la categoría de decisión para el pensamiento postestructuralista (en particular para su noción de sujeto) es notablemente escasa la atención que a ella se ha destinado. En nuestra concepción, la decisión es un momento de la subjetividad previo al sujeto que tiene función instituyente.

(14) En otra tradición esta instancia de disrupción de la lógica de la repetición y las condiciones para la aparición y la novedad es recuperado por Hannah Arendt. Allí las distinciones liberación y libertad, poder y violencia, juegan un papel importante en la concepción de la acción política.

(15) La presentación de aportes para una teoría de los sujetos sociales supera los límites del presente trabajo. Nos basta con una definición provisoria y acotada de sujetos sociales como configuraciones subjetivas colectivas estables y con rasgos identitarios involucradas directamente en los procesos de dar sentido a situaciones, acontecimientos o relaciones sociales. Para una discusión sobre las teorías de los actores colectivos, ver Estrada 1997.

(16) Esta característica hace que autores como Luhmann (Torres 2004) se refieran a los movimientos sociales como movimientos de protestas, asignándoles la función de elaborar demandas hacia el sistema político para que éste lo procese de acuerdo a su lógica.

(17) Un tratamiento exhaustivo de ciertas implicaciones del concepto de demanda en Laclau puede encontrarse en el notable trabajo de Carassale (2007). 
(18) Un debate entre Lacan y Castoriadis tiene como objeto determinar la primacía de la falta sobre el deseo o viceversa (Elliot 1995).

(19) La referencia a la vinculación de la protesta social con el espacio público ha sido trabajada por Naishtat (1999 у 2005).

(20) Esto implica, como argumenta Muñoz (2006) concebir que hay demandas que se ubican, en principio, fuera de lo que Rancière concibe como lógica de la igualdad.

(21) Con Laclau y Mouffe (1985) nos referimos a las "posiciones de sujeto" para identificar la ubicación de un sujeto en la trama de relaciones sociales que componen el orden social.

(22) La situación de no contar con agua potable, servicios sanitarios, salud y educación puede considerarse como una situación desventajosa, pero no por ello necesariamente espacio de una demanda concreta.

(23) El pedido de un semáforo o la extensión de la red de agua potable bien puede ser absorbido diferencialmente por la gestión del orden (el municipio), sin embargo problemas burocráticos, ineficiencias o especulaciones políticas pueden conducir a que no se satisfaga el reclamo. Esto abre diferentes posibilidades, tanto la construcción de un campo fértil para la expansión y articulación de otras demandas, la construcción de una alteridad ("el gobierno que incumple sus deberes") como la cancelación de la demanda por el desaliento ("es inútil, nadie nos atiende"). Es esto lo que hay que estudiar en cada caso y allí la historicidad del orden, la historia de las relaciones sociales, sus actores e instituciones, sus imaginarios, las experiencias ancladas en la memoria histórica, etc., serán aspectos fundamentales.

(24) Aboy (2005:117) repara en que lo que se vuelve vacío es el significado. Es decir, un significado que llena particularmente un significante se amplia para capturar otros significados que tienen como referencia al mismo significante.

(25) La centralidad de los significados sociales en la definición de la subjetividad colectiva (y la intervención de ésta en la elaboración de la demanda) hace que el campo vinculado a los sentidos sea una referencia ineludible a la hora de planear investigaciones de los movimientos sociales y sus demandas. En lo que concierne a lo metodológico, el cruce de técnicas en el trabajo de campo es fundamental como forma de iluminar diferentes aspectos del proceso histórico objetivado. En efecto, mediante observación es posible realizar reconstrucciones de los sentidos a partir de los signos (creación, movilización, reproducción puestos en juego en las interacciones en espacios sociales). Mientras que, por otro lado, a través de diferentes técnicas de entrevistas es posible buscar una reconstrucción del sentido atribuido por los propios sujetos. Este doble afluente confluye en el abordaje propio de la tradición etnográfica que diversos autores han recuperado recientemente (Ameigeiras 2006). Asimismo se vincula con los desarrollos contemporáneos de análisis del discurso que se ocupan de indagar en las prácticas productores de sentido tanto lingüísticas como no lingüísticas de Van Dijk (1999). 


\section{Bibliografía}

Aboy, G. 2005. Identidad y diferencia política. En: Schuster, F. et. al. Tomar la palabra. Estudios sobre protesta social y acción colectiva en la Argentina contemporánea. Buenos Aires: Prometeo, pp. 111-128.

Ameigeiras, A. 2006. El abordaje etnográfico en la investigación social. En: Vasilachis de Gialdino (coord.) Estrategias de investigación cualitativa. Barclona: Gedisa.

Carassale, S. 2007. La demanda de la demanda. La mirada del espectro. En Aibar, J. (comp.) Vox populi. Populismo y democracia en América Latina. México: FLACSO.

Castoriadis, C. 1986. El campo de lo social histórico. Estudios filosofía / historia / letras 4, artículo 1. http://biblioteca.itam.mx/estudios/estudio/estudio04/sec_3.html

Castoriadis, C. 2007. La institución imaginaria de la sociedad. Buenos Aires: Tousquest.

Chihu, A. 2000. El análisis cultural de los movimientos sociales. Sociológica 15(42): 209-230.

Craig, J. 1994. La teoría de la movilización de recursos y el estudio de los movimientos sociales. Zona abierta 69: 5-50.

De Ípola, E. 2000. Acción, decisión, sujeto. Fractal 19(V): 79-98.

De la Garza, E. 2001. Subjetividad, cultura y estructura. Revista Iztapalapa 50: 83-104.

Santos, B. 2006. Renovar la teoría crítica y reinventar la emancipación social. Buenos Aires: CLACSO.

Dussel, E. 1998. Ética de la Liberación. En la edad de la globalización y de la exclusión. Madrid: Trotta.

Dussel, E. 2006. 20 tesis de política. México: Siglo XXI-Crefal.

Eisinger, P. 1973. The conditions of protest behaviour in American cities. American political science review 67: $11-28$.

Elliot, A. 1995. Teoría social y psicoanálisis en transición. Sujeto y sociedad de Freud a Kristeva. Buenos Aires: Amorrortu.

Estrada, M. 1997. Es reformable la teoría de los actores colectivos? Revista mexicana de Sociología 59(3): 5579.

Foucault, M. 1991. Espacios de poder. Madrid: La Piqueta.

Giménez, G. 1994. Los movimientos sociales: problemas teórico-metodológicos. Revista mexicana de sociología 2: 3-24.

Hegel, G. 1992. Fenomenología del Espíritu. México: Fondo de Cultura Económica.

Jameson, F. 1995. Lo imaginario y lo simbólico en Lacan. Buenos Aires: El cielo por asalto. 
Klandermans, B. y Johnson, H. 1995. Social movements and culture. Minneapolis: University of Minnesota Press.

Laclau, E. 2005. La razón populista. Buenos Aires: Fondo de Cultura Económica.

Laclau, E. 2005b. Populismo: ¿Qué hay en el nombre? En: Arfuch, L. Pensar este tiempo. Espacios, afectos, pertenencias. Buenos Aires: Paidós, pp. 25-46.

Laclau, E. 2000. Nuevas reflexiones sobre la revolución en nuestro tiempo. Buenos Aires: Nueva Visión.

Laclau, E. 1996. Emancipación y diferencia. Buenos Aires: Ariel.

Laclau, E. 1985. Tesis acerca de la forma hegemónica de la política. En: Martín Del Campo, J.L. Hegemonía y alternativas políticas en América Latina. México: Siglo XXI, pp. 19-44.

Laclau, E. y Mouffe, C. 2004. Hegemonía y estrategia socialista. Buenos Aires: Fondo de Cultura Económica.

Laclau, E. y Mouffe, C. 1985. Hegemony and socialist strategy. Towards a radical democratic politics. London: Verso.

Laraña, E. y Gusfield, J. 1994. Los nuevos movimientos sociales: de la ideología a la identidad. Madrid: CIS.

Lefort, C. 1991. Ensayos sobre lo político. Guadalajara: Ediciones Universidad de Guadalajara.

Luhmann, N. 1996. Introducción a la Teoría de Sistemas. Lecciones publicadas por Javier Torres Nafarrate. México: Universidad Iberoamericana.

Marchart, O. 2009. El pensamiento político postfundacional. La diferencia política en Nancy, Lefort, Badiou y Laclau. Buenos Aires: Fondo de Cultura Económica.

McCarthy, J. y Zald, M. 1977. Resource mobilization and social movements: A partial theory. American journal of sociology 86(6): 1212-1241.

McAdam, D. 1994. Cultura y movimientos sociales. En: Laraña, E. y Gusfield, J. Los nuevos movimientos sociales. De la ideología a la identidad. Madrid: CIS, pp. 43-68.

McAdam, D., Tarrow, S. y Tilly, C. 2001. Dynamics of contention. Cambridge: Cambridge University Press.

McAdam, D., McCarthy, J. y Zald, M. 1999. Perspectivas comparadas: oportunidades políticas, estructuras de movilización y marcos interpretativos culturales. Madrid: Istmo.

Melucci, A. 1999. Acción colectiva, vida cotidiana y democracia. México: El Colegio de México.

Melucci, A. 1994. Asumir un compromiso: identidad y movilización en los movimientos sociales. Zona abierta 69: 153-180.

Munck, G. 1995. Algunos problemas conceptuales en el estudio de los movimientos sociales. Revista mexicana de sociología 3: 17-39. 
Muñoz, M.A. 2006. Laclau y Rancière: algunas coordenadas para la lectura de lo político. Andamios: revista de investigación social 4: 119-144.

Naishtat, F. 2005. Ética pública de la protesta colectiva. En: Schuster, F. et. al. Tomar la palabra. Estudios sobre protesta social y acción colectiva en la Argentina contemporánea. Buenos Aires: Prometeo, pp. 25-42

Naishtat, F. 2004. Problemas filosóficos en la acción individual y colectiva. Una perspectiva pragmática. Buenos Aires: Prometeo.

Naishtat, F. 1999. Acción colectiva y regeneración democrática del espacio público. En: Quiroga, H., Villavicencio, S. y Vermeren, P. Filosofías de la ciudadanía. Sujeto político y democracia. Rosario: Homo Sapiens.

O'Donnell, G. 1978. Apuntes para una teoría del Estado. Revista Mexicana de Sociología 40(4): 1157-1199.

Rancière, J. 1996. El desacuerdo. Filosofía y política. Buenos Aires: Nueva Visión.

Retamozo, M. 2006a. Esbozos para una epistemología de los sujetos y movimientos sociales. Cinta moebio 26: 1-16.

Retamozo, M. 2006b. Los piqueteros: Trabajo, subjetividad y acción colectiva en el movimiento de desocupados en Argentina. América Latina hoy 42: 109-128.

Saltalamacchia, H. 1987. Historia de vida y movimientos sociales: el problema de la representatividad. Revista Mexicana de Sociología 49(1): 255-277.

Saltalamacchia, H., Colon, H. y Rodriguez, H. 1983. Historias de vida y movimientos sociales: propuestas para el uso de la técnica. Revista de Iztapalapa 9: 321-338.

Schuster, F. et. al. 2005. Tomar la palabra. Estudios sobre protesta social y acción colectiva en la Argentina contemporánea. Buenos Aires: Prometeo.

Schuster, F. y Pereyra, S. 2001. La protesta social en la Argentina democrática: balance y perspectiva de una acción política. En: Giarracca, N. (y colab.) La protesta social en Argentina. Buenos Aires: Alianza.

Stravakakis, Y. 2007. Lacan y lo político. Buenos Aires: Prometeo-UNLP.

Tarrow, S. 1997. El poder en movimiento. Los movimientos sociales, la acción colectiva y la política. Madrid: Alianza Universidad.

Torres, J. 2004. Luhmann: la política como sistema. México: FCE-Universidad Iberoamericana- UNAM.

Touraine, A. 1991. Los movimientos sociales. Buenos Aires: Almagesto.

Touraine, A. 1990. Movimientos sociales de hoy. Actores y analistas. Barcelona: Editorial Hacer.

Van Dijk, T. 1999. El análisis crítico del discurso. Anthropos 186: 23-36. 
Villasante, T. 1994. De los movimientos sociales a las metodologías participativas. En: Delgado, J. y Gutierrez, J. Métodos y técnicas cualitativas de investigación en ciencias sociales. Madrid: Síntesis, pp. 399-426.

Zemelman, H. 1987. Conocimiento y sujetos sociales. México: El Colegio de México.

Zizek, S. 1998. Porque no saben lo que hacen. El goce como un factor político. Paidós: Espacios del Saber.

Recibido el 29 Abril 2009

Aceptado el 14 Jul 2009 